\title{
Review of: "A four-season study quantifying the weekly external training loads during different between match microcycle lengths in professional rugby league"
}

João PAulo Reis Gonçalves Moreira de Brito ${ }^{1}$

1 Instituto Politécnico de Santarém

Potential competing interests: The author(s) declared that no potential competing interests exist.

Dear Editor,

I appreciate the opportunity to review the manuscript entitled "A four-season study quantifying the weekly external training loads during different between match microcycle lengths in professional rugby league". The study aim was to quantify and compare external training load metrics between different betweenmatch microcycle lengths. A secondary aim was to determine total weekly training load variation due to microcycle, individual and head coach.

The manuscript addresses a relevant issue that has not been studied much to date. The manuscript is well structured and written, clearly presenting the methodology and discussing the results clearly.

For that reason I have only minor change suggestions that I point out below for the authors. In my opinion, the manuscript is ready to be accepted for publication.

\# In figure 1 there are no $P<0.001$. It is suggested to remove the 3 asterisks from the figure caption. Having no further considerations to make about the manuscript, I congratulate the authors for the good work done.

Best Regards

João Paulo Brito 